\title{
GC content but not nucleosome positioning directly contributes to intron-splicing efficiency in Paramecium
}

Stefano Gnan ${ }^{1,4}$, Mélody Matelot ${ }^{2,4}$, Marion Weiman $^{3}$, Olivier Arnaiz ${ }^{3}$, Frédéric Guérin ${ }^{2}$, Linda Sperling ${ }^{3}$, Mireille Bétermier ${ }^{3}$, Claude Thermes ${ }^{3}$, Chun-Long Chen ${ }^{1 *}$ and Sandra Duharcourt ${ }^{2}$ *

1 Institut Curie, Université PSL, Sorbonne Université, CNRS UMR3244, Dynamics of Genetic Information, Paris, 75005, France

2 Université de Paris, CNRS, Institut Jacques Monod, F-75006, Paris, France

${ }^{3}$ Université Paris-Saclay, CEA, CNRS, Institute for Integrative Biology of the Cell (I2BC), 91198, Gifsur-Yvette, France

${ }^{4}$ The authors wish it to be known that, in their opinion, the first 2 authors should be regarded as joint First Authors.

* To whom correspondence should be addressed.

Sandra Duharcourt, Tel: [+33-(0)157278009]; Email: [sandra.duharcourt@ijm.fr]

ORCID: https://orcid.org/0000-0002-8913-8799

Chun-Long Chen, Tel: [+33-(0)156246205]; Fax: [+33-(0)156246674]; Email: [chunlong.chen@curie.fr] ORCID: https://orcid.org/0000-0002-4795-0295

Present Address:

[Mélody Matelot], IGBMC - CNRS UMR 7104 - Inserm U 1258, 1 rue Laurent Fries, BP 10142, 67404 Illkirch CEDEX, France

[Marion Weiman], OncoDNA group, IntegraGen, Evry, 91000, France

[Frédéric Guérin], Scipio bioscience, 92120 Montrouge, France 


\section{ABSTRACT}

Eukaryotic genes are interrupted by introns that must be accurately spliced from mRNA precursors. With an average length of $25 \mathrm{nt}$, the $>90,000$ introns of Paramecium tetraurelia stand among the shortest introns reported in eukaryotes. The mechanisms specifying the correct recognition of these tiny introns remain poorly understood. Splicing can occur co-transcriptionally and it has been proposed that chromatin structure might influence splice site recognition. To investigate the roles of nucleosome positioning in intron recognition, we determined the nucleosome occupancy along the $P$. tetraurelia genome. We showed that $P$. tetraurelia displays a regular nucleosome array with a nucleosome repeat length of $\sim 151 \mathrm{bp}$, amongst the smallest periodicities reported. Our analysis revealed that introns are frequently associated with inter-nucleosomal DNA, pointing to an evolutionary constraint to locate introns at the AT-rich nucleosome edge sequences. Using accurate splicing efficiency data from cells depleted for the nonsense-mediated decay effectors, we showed that introns located at the edge of nucleosomes display higher splicing efficiency than those at the centre. However, multiple regression analysis indicated that the $\mathrm{GC}$ content, rather than nucleosome positioning, directly contributes to intron splicing efficiency. Our data reveal a complex link between GC content, nucleosome positioning and intron evolution in Paramecium.

Keywords: Nucleosome positioning, intron splicing, GC content, Nonsense Mediated Decay, Paramecium 


\section{INTRODUCTION}

In eukaryotes, genomic DNA is compacted by histones into chromatin. The basic unit of chromatin is the nucleosome, which comprises a histone octamer made of the four core histones $(\mathrm{H} 2 \mathrm{~A}, \mathrm{H} 2 \mathrm{~B}, \mathrm{H} 3$ and $\mathrm{H} 4)$ and 146-147 bp of DNA wrapped around it (1,2). Nucleosomes are not randomly located along the genome but positioned with respect to DNA sequence. The affinity of DNA for histone octamers and the energy needed to bend different DNA fragments around the histone octamer are influenced by the primary DNA sequence, which is therefore an important determinant of nucleosome positioning along the genome (3-9). Nucleosome positioning is highly dynamic and its regulation is crucial to control chromatin accessibility and recruitment of chromatin modifiers and transcription factors (10-13). In most genomes, genes display a Nucleosome Free Region (NFR) at the 5' of their Transcription Start Site (TSS) due to the formation of complexes made by transcription factors around promoter regions (1315). A second NFR is present at Transcription Termination Sites (TTS), likely due to the adverse nucleotide composition of the poly $(\mathrm{A})$ signal $(16,17)$. Nucleosomes are organized in regular arrays with a periodic distance called the nucleosome repeat length (NRL). Such periodicity is especially evident over gene bodies, and it is species and cell-type specific $(18,19)$. Genome-wide studies have shown that nucleosomes are preferentially positioned in exons compared to introns in diverse organisms including Schizosaccharomyces pombe, Drosophila, worms and human (20-25). Several lines of evidence indicated that a well-positioned nucleosome might slow down RNA polymerase II and favour exon inclusion and alternative splicing $(26,27)$, suggesting a functional role of nucleosome arrays during mRNA maturation. This is in agreement with recent studies showing that intron splicing can occur in a co-transcriptional manner $(28,29)$. Some studies have suggested that GC richness at exons, and not nucleosome positioning per se, is important for intron splicing $(30,31)$. Yet, the contribution of nucleosome positioning to intron splicing efficiency has not been investigated thoroughly. The nonsense-mediated decay (NMD) machinery recognizes and degrades transcripts containing premature termination codons $(32,33)$. Therefore, most of the mis-splicing or un-splicing events are removed rapidly by this powerful surveillance mechanism to avoid the production of erroneous proteins. To date, most studies estimated splicing efficiency from NMD-proficient cells, which eliminate most missplicing or un-splicing events, and therefore cannot provide a solid evaluation of intron splicing efficiency.

The ciliate Paramecium tetraurelia is a unicellular eukaryotic model organism. Like all ciliates, two distinct types of nuclei co-exist within the same cytoplasm in P. tetraurelia (34). The diploid germline micronucleus (MIC) is transcriptionally silent during vegetative growth and transmits the germline genome to sexual progeny through meiosis, while the highly polyploid somatic macronucleus (MAC) is responsible for gene expression (35). The $>90,000$ introns annotated in the MAC genome are among the shortest reported in eukaryotes (18 to $33 \mathrm{nt}, 25 \mathrm{nt}$ on average) (36). How such a large number of tiny introns can be efficiently spliced is not known. In $P$. tetraurelia, no alternative splicing has been reported so far and introns are associated with weak splice signals. A strong counter-selection for introns that cannot be detected by the NMD machinery was previously shown, suggesting that introns 
rely on NMD to compensate for suboptimal splicing efficiency and accuracy $(36,37)$. Whether nucleosome positioning or other factors, such as GC content, can regulate splicing efficiency and shape intron evolution in Paramecium has not been studied so far.

Here, we investigated a possible role of nucleosome positioning in the recognition of introns in $P$. tetraurelia. We mapped the nucleosome occupancy in the somatic nuclei through paired-end MNaseseq. We found that $P$. tetraurelia displays a regular nucleosome array along genes, with a nucleosome repeat length of $\sim 151 \mathrm{bp}$, amongst the smallest periodicities reported in eukaryotes. We compared the positioning of nucleosomes with that of introns and observed that introns are frequently located at the edges of nucleosomes, i.e. associated with linker sequences. Using the accurate splicing efficiency data determined from NMD-depleted cells, we show that introns located at the edge of nucleosomes display higher splicing efficiency than those at the centre. Multiple regression analysis revealed that this difference in splicing efficiency is not explained by the nucleosome positioning per se but rather by the fact that the sequences at the edges of nucleosomes are more AT-rich. This study allowed us to reveal a complex link between nucleosome positioning, GC content and intron splicing efficiency. We propose a model in which a selection constraint during Paramecium genome evolution has displaced nucleosome positioning relative to introns, so that the intron sequences are frequently located at the AT-rich edge of nucleosomes to favour efficient splicing.

\section{MATERIAL AND METHODS}

\section{Paramecium strains, cultivation and autogamy}

All experiments were carried out with the entirely homozygous wild type strain 51 of $P$. tetraurelia. Cells were grown at $27^{\circ} \mathrm{C}$ in wheat grass powder (WGP) infusion medium bacterized the day before use with Klebsiella pneumoniae and supplemented with $0.8 \mathrm{mg} / \mathrm{mL} \beta$-sitosterol $(38,39)$.

\section{Macronuclei preparation}

Cells were exponentially grown for 12 divisions then cultures at 1,000 cells $/ \mathrm{mL}$ were filtered through eight layers of sterile gauze. Cells were collected by low-speed centrifugation (550 g for $1 \mathrm{~min}$ ) and washed once with $10 \mathrm{mM}$ Tris- $\mathrm{HCl} \mathrm{pH} \mathrm{7.4}$. The pellet was diluted 3-fold by addition of lysis buffer $(0.25$ M sucrose, $10 \mathrm{mM} \mathrm{MgCl} 2,10 \mathrm{mM}$ Tris pH 6.8, $0.2 \%$ Nonidet P-40) and processed at $4^{\circ} \mathrm{C}$ as described in (40) with some modifications. Briefly, cells were lysed with 10 strokes of a Dounce homogenizer. Particular care was taken to make sure that macronuclei were still intact under the microscope. Washing buffer $(0.25 \mathrm{M}$ sucrose, $10 \mathrm{mM} \mathrm{MgCl}, 10 \mathrm{mM}$ Tris-HCl pH 7.4) was added to a final volume of 10 times the initial pellet. Macronuclei were collected by centrifugation at 2,000 $\mathrm{g}$ for $1 \mathrm{~min}$ and washed once in washing buffer. The pellet was diluted 2-fold in $2.1 \mathrm{M}$ sucrose, $10 \mathrm{mM} \mathrm{MgCl}$, $10 \mathrm{mM} \mathrm{Tris} \mathrm{pH} 7.4$ and loaded on top of a 3-mL sucrose layer (2.1 M sucrose, $10 \mathrm{mM} \mathrm{MgCl}, 10 \mathrm{mM}$ Tris- $\mathrm{HCl} \mathrm{pH} \mathrm{7.4)} \mathrm{and}$ centrifuged in a swinging rotor for $1 \mathrm{hr}$ at $210,000 \mathrm{~g}$. The macronuclear pellet was washed once, centrifuged at $2,000 \mathrm{~g}$ for $1 \mathrm{~min}$ and resuspended in washing buffer at $10^{7}$ nuclei/ $/ \mathrm{mL}$. The macronuclei recovery is quite low, of the order of $10-20 \%$. 


\section{MNase digestion on chromatin isolated from macronuclei}

Samples containing $10^{5}$ macronuclei were incubated in the digestion buffer $(0.25 \mathrm{M}$ sucrose, $10 \mathrm{mM}$ $\mathrm{MgCl}_{2}, 10 \mathrm{mM}$ Tris $\mathrm{pH} 7.4,1 \mathrm{mM} \mathrm{CaCl}$ ) with increasing amounts $(0,0.5,1,2,5,7.5,10 \mathrm{U})$ of MNase (Sigma) at $30^{\circ} \mathrm{C}$ for $10 \mathrm{~min}$. Reactions were stopped by the addition of 3 volumes of $0.5 \mathrm{M} \mathrm{EDTA} \mathrm{pH}$ 9.0, $1 \% \mathrm{~N}$-laurylsarcosine (Sigma), $1 \% \mathrm{SDS}, 1 \mathrm{mg} / \mathrm{mL}$ proteinase $\mathrm{K}$ (Merck) and incubated at $55^{\circ} \mathrm{C}$ overnight. DNA from each sample was gently extracted once with phenol, and dialyzed twice against TE (10 mM Tris-HC1, $1 \mathrm{mM}$ EDTA at pH 8.0) containing $25 \%$ ethanol, and once against TE. Samples were then treated with RNase A and DNA was quantified with a Nanodrop spectrophotometer (Thermo Scientific) and separated on a $1.2 \%$ agarose gel. The reactions containing mostly mono-nucleosomal DNA fragments (see Fig. 1) were selected and mono-nucleosomal DNA fragments were purified from $3 \%$ low melting-temperature agarose gels and treated with $\beta$-agarase (Sigma) for sequencing.

\section{MNase digestion on naked DNA}

Following purification on a sucrose layer, the macronuclear pellet was washed once, centrifuged at $2,000 \mathrm{~g}$ for $1 \mathrm{~min}$, and was resuspended in three volumes of lysis solution (0.5 M EDTA at pH 9.0, 1\% SDS, $1 \% \mathrm{~N}$-laurylsarcosine (Sigma), $1 \mathrm{mg} / \mathrm{mL}$ of proteinase $\mathrm{K}$ (Merck) then incubated at $55^{\circ} \mathrm{C}$ overnight. DNA was gently extracted with phenol, and dialyzed twice against TE (10 mM Tris-HC1, 1 mM EDTA at $\mathrm{pH} 8.0$ ) containing $20 \%$ ethanol, and once against Tris $10 \mathrm{mM} \mathrm{pH}$ 8.0. 1.6 $\mu \mathrm{g}$ of DNA was digested with increasing amounts of MNase $\left(0\right.$ to $\left.1 \times 10^{-3} \mathrm{U}\right)$ in the digestion buffer at $30^{\circ} \mathrm{C}$ for $10 \mathrm{~min}$. The reactions were stopped with $250 \mathrm{mM}$ EDTA. The samples were analysed on a $1.2 \%$ agarose gel and reactions containing fragments of 100-200 bp were gel-purified for DNA sequencing (see Fig. S1).

\section{MNase library preparation and sequencing}

Sequencing libraries were generated using the sequencing kit: TruSeq SBS Kit v5 - GA (36 Cycle) (FC104-5001, Illumina). Samples were then sequenced on an Illumina GA-Ilx sequencer using paired-end (PE) 74 bp setting. Alignment was performed using ELAND and mapping to the MAC genome of strain 51 v1.0 (ptetraurelia_mac_51.fa), available at ParameciumDB (https://paramecium.i2bc.parissaclay.frl) (41).

\section{Nucleosome positioning calling}

After aligning reads to the reference MAC genome, PCR duplicates with the same start and end positions were removed. Only reads from mono-nucleosomes were kept, therefore, read pairs longer than $150 \mathrm{bp}$ and shorter than 75 were excluded. We used only the data within the scaffolds larger than $200 \mathrm{~kb}$. A nucleosome score was calculated using the central $75 \mathrm{bp}$ of each read pair. Signal was then smoothed with a gaussian filter and a sigma of 30 over $90 \mathrm{bp}$ for visual assessment of nucleosome position calling. Local Maxima and local Minima were identified by convoluting the nucleosome score with a first derivative of a gaussian (sigma 30 over $\pm 90 \mathrm{bp}$ ). The points of inflection were identified by convoluting the nucleosome score with a kernel containing the second derivative of a gaussian (sigma 30 over $\pm 90 \mathrm{bp}$ ). Peaks were called as a local maximum between two inflection points with opposite inclination. Peaks were called independently in the two chromatin samples, and then a list of well- 
positioned nucleosomes was compiled using those nucleosomes whose dyad (i.e. centre) differs by less than 10 bp between the two biological replicates ( $80 \%$ of all nucleosomes). These well-positioned nucleosomes were used for the downstream analyses.

\section{Computation of Nucleosome Repeat Length}

To compute the Nucleosome Repeat Length (NRL), we first calculated the distance of each nucleosome to all the other nucleosomes on the same scaffold, then used the distances obtained to generate the density distribution. This density distribution was then smoothed using a gaussian filter (sigma=10 over $\pm 30 \mathrm{bp}$ ) and local maxima identified convoluting the density distribution with the first derivative of a gaussian (sigma $=10$ over $\pm 30 \mathrm{bp}$ ). The first 8 local maxima were then ordered by increasing distances and fitted using a linear model. The slope of the fitted model corresponds to the NRL.

\section{Nucleosome distribution calculation}

Gene annotation v2.0 of MAC was from (41), and the transcription start sites and transcription termination sites were from (42). To compare with the distribution of real exon sizes, a set of simulated exons was created assuming uniform exon sizes within each gene, i.e. for a given gene with $n$ exons, we divided its total exon length by $n$ to get the length of $n$ simulated exons of the corresponding gene. The NMD data were obtained from (37), splicing efficiency of each intron was calculated as the Splicing events / Total number of observations (i.e. spliced + unspliced reads). Mean profiles and heatmaps were drawn using a customized script and plotting using matplotlib. All statistical analyses were performed with Python (version 3.7.4).

\section{Multilinear regression}

Starting parameters used for the multiple linear regression can be found in Table S1. Parameters were transformed using appropriate functions in order to maximise their linearity with the intron splicing efficiency, e.g. log transformation of expression levels. Values were then standardized. A randomly selected set of introns ( $10 \%$ of all introns) was kept from the multilinear regression model fitting, and used as a test dataset to evaluate the model performance. Parameters were tested in multiple combinations. For each combination, after each fitting, we performed a two-sided Z-test per each coefficient with $\mathrm{H}_{0}: \mathrm{C}=0$ and $\mathrm{H}_{1}: \mathrm{C} \neq 0$. Statistically significant coefficients were then retained and the linear model was trained again with the associated parameters. This step was repeated until the number of variables stabilised. Using the intron test dataset, we calculated the Pearson correlation between real and predicted data, and the best predicting model was kept. Estimation of the contribution of each parameter is calculated as in (43), which is based on the absolute value of the product of each coefficient and the Pearson correlation value of its parameter with the splicing. Contributions were then converted to percentages. 


\section{RESULTS}

\section{Genome-wide nucleosome position profiling along the Paramecium somatic genome}

Using MNase-seq, we derived a first nucleosome positioning profile of the macronuclear (MAC) genome of $P$. tetraurelia during vegetative growth. Both chromatin samples and naked MAC DNA controls were digested to mono-nucleosome size ( 150 bp, Fig.1 A \& B and Fig. S1 A). The results obtained from two biological replicates were highly reproducible (Pearson $\mathrm{R}=0.92$, Fig. S1 B). We therefore combined data from both biological replicates for downstream analyses. All the data presented in the main figures were obtained with the average of two chromatin samples and two naked DNA controls, respectively. The results of each individual sample are reported in the supplementary figures. Using the gene annotation, together with the Transcription Start Sites (TSSs) identified by 5 ' CAP-seq and Transcription Termination Sites (TTSs) identified by poly(A) detection (42), we investigated nucleosome occupancy along transcription units and around their extremities. As described in other eukaryotes, $P$. tetraurelia presents an enriched nucleosome density over the transcription units compared to the flanking regions, showing regular arrays of nucleosomes over transcription units (Fig. 1 C-D and Fig. S1 C-D). As expected, we were able to identify Nucleosome Free Regions (NFRs) upstream of the TSSs of Paramecium genes, followed by an array of well-positioned nucleosomes (Fig. 1 C-D and Fig. S1 C). The analysis of TTSs shows regions with very low nucleosome occupancy downstream of the TTSs and a weakly organised array towards the gene body (Fig. $1 \mathrm{D}$ and Fig. S1 D). We further separated gene pairs into 3 groups based on their disposition: tandem $(n=20,298)$, convergent $(n=8,900)$ and divergent $(n=8,890)$ (Fig. 1 E and Fig. S1 E-G). Interestingly, we found that nucleosome arrays are clearly visible upstream of the TTS only when genes are positioned in tandem or divergent pairs (Fig. $1 \mathrm{E}$ and Fig. S1 F), but not in convergent pairs (Fig. $1 \mathrm{E}$ and Fig. S1 G). This observation suggests that the nucleosome positioning at TTS observed for tandem genes might be due to the downstream TSS, as suggested for Saccharomyces cerevisiae (44). Alternatively, convergent genes might be influenced by the transcription readthrough of the gene in the opposite orientation.

Based on our nucleosome position calling and using only well-positioned nucleosomes identified in both replicates (see Materials and Methods), we calculated the nucleosome repeat length (NRL) (Materials and Methods). We found that $P$. tetraurelia displays one of the smallest NRL reported in eukaryotes $(150.79 \pm 0.56$ bp on average, Fig. $1 \mathrm{~F}-\mathrm{G}$ and Fig. S1 H-I), close to the $156 \pm 2$ bp of S. pombe (45), which is much smaller than the $165 \mathrm{bp}$ of $S$. cerevisiae (46). In human, the NRL within gene bodies is smaller than outside (47). We performed a similar analysis sub-setting nucleosomes based on whether their centres overlap with gene bodies or not. We found a negligible difference between the NRL within gene bodies $(150.79 \pm 0.70 \mathrm{bp}$, more than $80 \%$ of the analysed sequences) and those outside of genes (150.99 \pm 1.17 bp) (Fig. S1 J). 
A

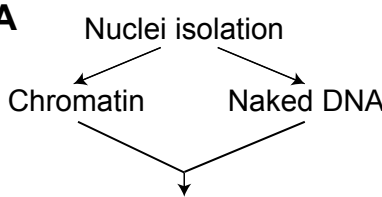

B MNase

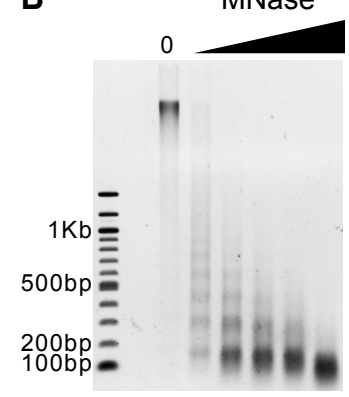

C

MNase digestion

DNA purification

DNA-seq

Chromatin

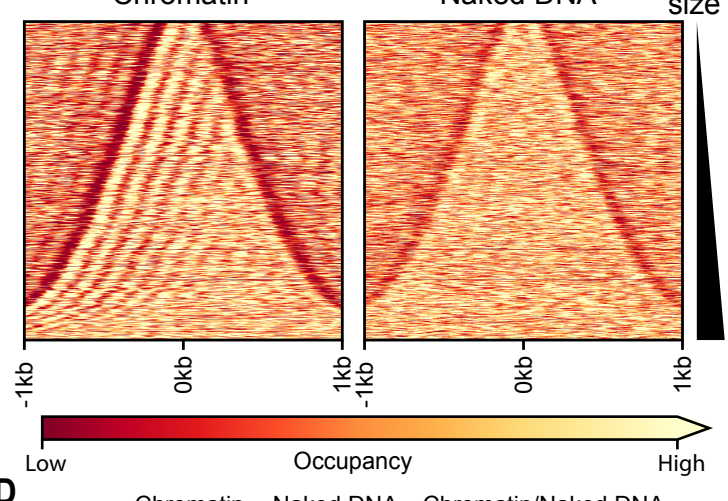

D

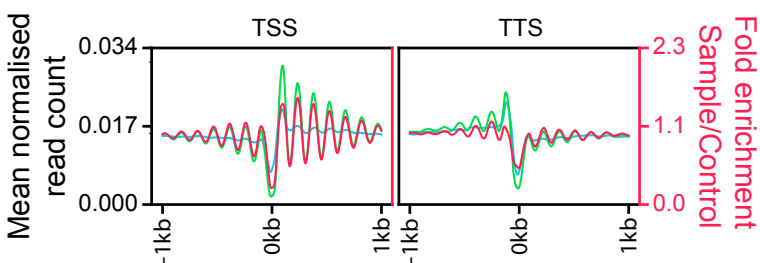

E -Chromatin - Naked DNA-Chromatin/Naked DNA

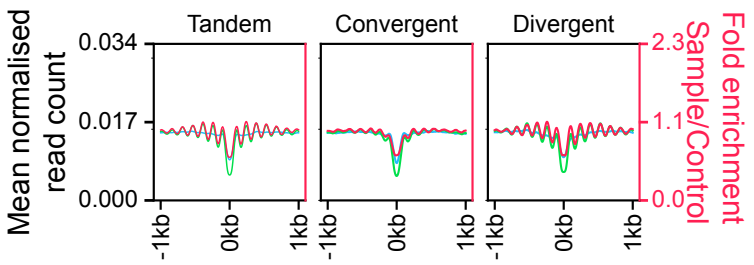

$\mathbf{F}$

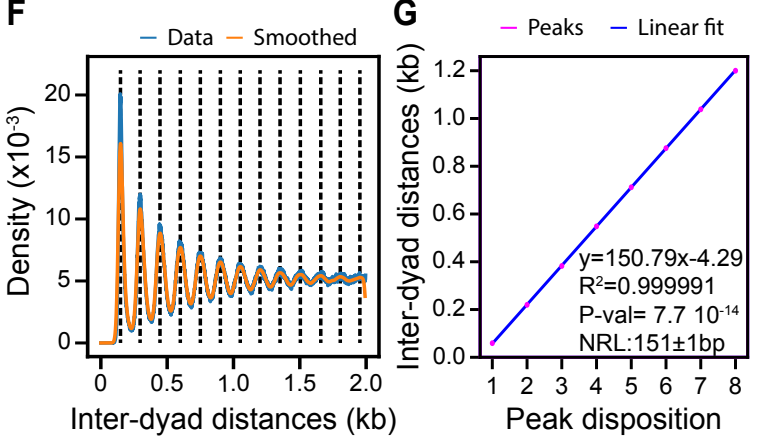

Figure 1. Nucleosome occupancy along the Paramecium MAC genome. (A) Schematic representation of the MNase-seq experiment. (B) MNase digestion of MAC chromatin with increasing MNase enzyme concentration. (C) Heatmap showing nucleosome occupancy $\pm 1 \mathrm{~kb}$ around the centre of each gene ordered by gene size (small genes on the top and large genes on the bottom) for 38,256 genes located on scaffolds that are at least $200 \mathrm{~kb}$ long. Left panel: average of two chromatin treated samples (Chromatin). Right panel: average of two naked DNA control samples (Naked DNA). 
Average nucleosome occupancies around Transcription Start Sites (TSSs) identified by 5' CAP-seq on the left, and Transcription Termination Sites (TTSs) identified by poly(A) detection on the right: in green, the average profile of chromatin treated sample (Chromatin); in blue, average profile of naked DNA treated sample (Naked DNA); and in red the Chromatin/Naked DNA ratio, enrichment of which is shown on the second axis on the right (red axis). (E) Average nucleosome occupancies $\pm 1 \mathrm{~kb}$ around the centre of intergenic regions: same colour code as in panel D. Intragenic regions have been divided into three groups based on the relative positions of gene pairs: tandem (left), convergent (middle) or divergent (right). (F) Inter-centre distance between well-positioned nucleosomes (Materials and Methods) on the same scaffold. In blue, distance distributions from actual data (from $1 \mathrm{bp}$ to $2 \mathrm{~kb}$, binning=1); and in orange, the gaussian smoothed signal. Black dashed lines indicate the local maxima (peak centres) of the smoothed data (Materials and Methods). (G) In pink, the first 8 local maxima from Fig. $1 \mathrm{~F}$ ordered by increasing distance, and in blue the linear fitted model. At the bottom right, information about linear fitting and estimated NRL (Mean $\pm S D$ ) is given. P-value is calculated using a two-sided Z-test.

\section{The tiny introns of Paramecium genes are frequently associated with inter-nucleosomal DNA}

We then analysed nucleosome positioning over gene bodies. In $P$. tetraurelia, exons range from several nucleotides to a few kilobases (Fig. 2 A, Fig. S2 A) and are interspersed with tiny introns, spanning between 18 and 35 bp with a median size of 25 bp (Fig. 2 B). By visual inspection of the nucleosome occupancy profiles, we noticed a tendency of the MNase signal to be stronger over exons leaving the introns preferentially between two nucleosome peaks (Fig. 2 C, Fig. S2 B). This was especially visible when we examined the nucleosome density over introns sorted by the distance of each intron centre to the closest nucleosome centre (Fig. $2 \mathrm{D}$, Fig. S2 D). This distance is significant higher than what we would expect by calculating the distance of random positions inside gene bodies to the closest nucleosome centre ( $p$ value $<10^{-10}$ calculated with Mann-Whitney $U$ test, one sided, alternative $H_{1}$ : Introns distance from the closest nucleosome is higher than random chance. Fig. $2 \mathrm{E}$ ). Using this distance, we grouped introns into 3 categories: central, proximal and distal introns (as illustrated in Fig. $2 \mathrm{~F}$ ). We calculated their distribution and compared it with that of exons smaller than $300 \mathrm{bp}$ (roughly the same sample size) categorized in the same way (Fig. $2 \mathrm{G}$ ). Introns were found enriched at distal positions, i.e. located in the regions between two neighbour nucleosomes, compared to exons $(46 \%$ vs $22 \%$, respectively). In contrast, exons were more enriched in central positions compared to introns ( $47 \%$ vs $27 \%$, respectively). These distributions are statistically significantly different: $p$ value $<10^{-10}$ calculated with a $x^{2}$ test (Fig. $2 \mathrm{G}$ ). Moreover, $P$. tetraurelia exons seem to favour mono-nucleosome length sizes with $35 \%$ of exons having sizes comprised between $100 \mathrm{bp}$ and $200 \mathrm{bp}$. Such a size distribution is significantly shorter than what one would expect if exon sizes were simply uniformly distributed within each transcript (see Materials and Methods), in which case only $24 \%$ of the exons would fall in this range $\left(p<10^{-10}\right.$, Mann-Whitney $\mathrm{U}$ test, one sided, alternative $\mathrm{H}_{1}$ : simulated exons are bigger than real exons) (Fig. 2 A). Similar results were obtained using only exons of transcription units whose extremities are identified by both 5' CAP-seq and poly(A) detection (Fig. S2 A). This distribution of exon sizes might reflect some selective constraint to keep introns in phase in distal position, i.e. at the edge of the nucleosome. 
A

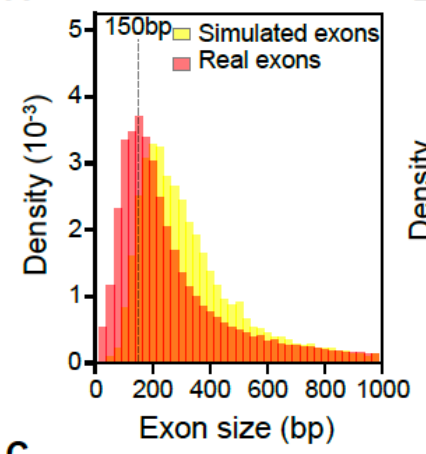

B

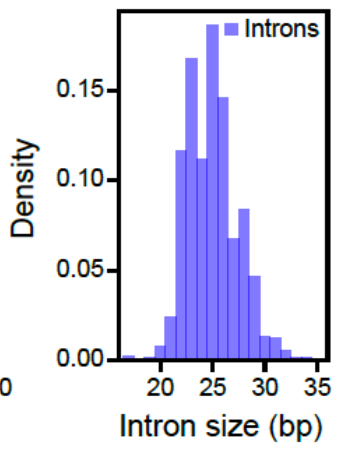

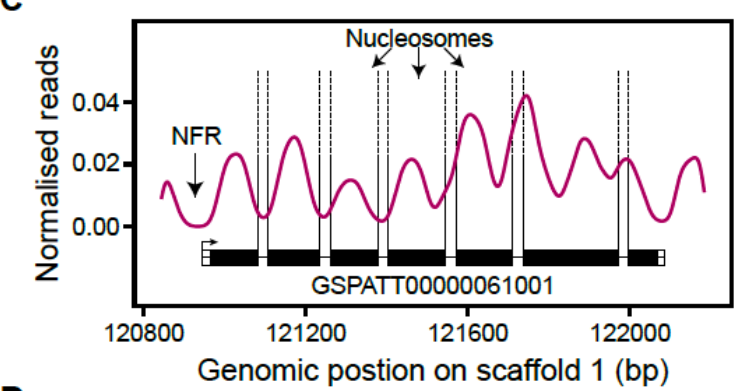

D

Chromatin

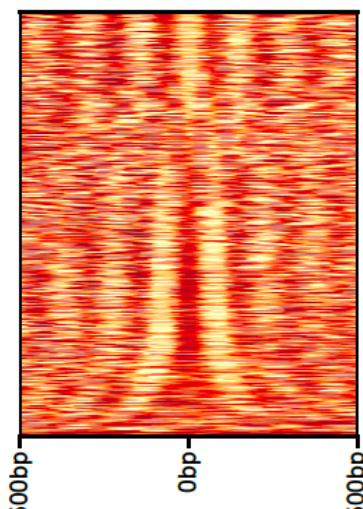

웅
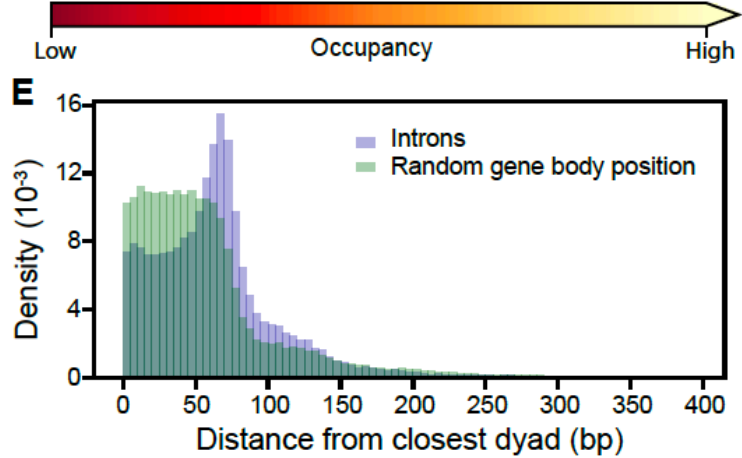

F

Proximal Proximal

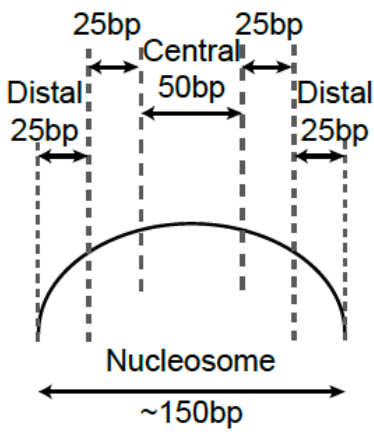

Naked DNA

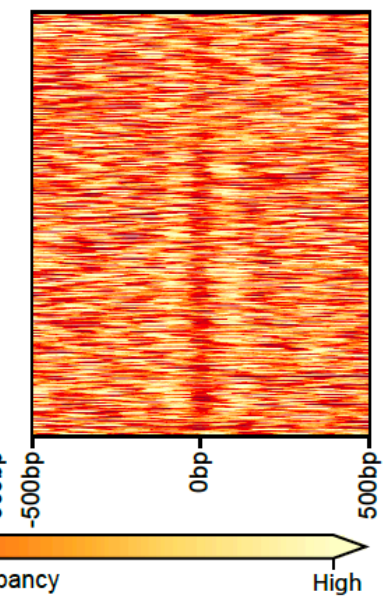

$\mathbf{G}$

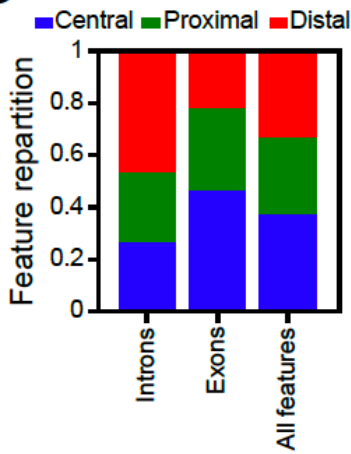


Figure 2. Inter-nucleosomal DNA is frequently associated with intron position. (A) Histogram showing exon size distribution (bin size $=25 \mathrm{bp}$ ): in red, real exons; and in yellow, simulated exons created assuming uniform exon sizes within each gene (see Materials and Methods). (B) Histogram showing intron size distribution (bin size $=1 \mathrm{bp}$ ). (C) Example track reporting nucleosome occupancy over genes with intron locations indicated by dashed vertical lines. We can observe nucleosome free regions (NFRs) around the gene promoters and introns frequently associated with inter-nucleosomal DNA. (D) Heatmap showing nucleosome occupancy \pm 500 bp around intron centres. Introns are ordered based on increasing distances from their centre to the closest nucleosome centre. The average of the chromatin samples is shown on the left and the average of the naked DNA samples on the right, with the same colour code as in Fig. 1 C. (E) Histogram reporting the distance of an intron centre to the closest nucleosome centre (blue). For each intron, a random position inside the corresponding gene body was selected and the distance to its closest nucleosome centre is reported (green). Bins size $=5$ bp. (F) Schematic representation of the criteria to assign features for each intron (or exon) into one of the 3 classes, based on the distance $(d)$ between its centre and the closest nucleosome centre position: central, $d \leq 25 \mathrm{bp}$; proximal, $25 \mathrm{bp}<d<50 \mathrm{bp}$; distal $50 \mathrm{bp} \leq d \leq 75 \mathrm{bp}$. (G) Relative distribution of introns, exons and both features over categories defined in $(F)$ for the introns overlapping with a fixed nucleosome (about $70 \%$ of all introns, see Materials and Methods) and exons with a size below $300 \mathrm{bp}$ overlapping with fixed peaks. See Fig. S2 C including the features with $d>75 \mathrm{bp}$.

\section{Higher splicing efficiency for introns at the edges of nucleosomes}

Previous studies have described the effect of nucleosome positioning on mRNA maturation in multiple organisms (20-25). To address whether nucleosome positioning affects intron splicing in $P$. tetraurelia, we examined the relationship between nucleosome positioning and intron splicing efficiency, using published datasets from both wild-type (WT) and NMD-depleted cells, which provide a measurement of the splicing efficiency of $P$. tetraurelia introns (37). Since NMD has been shown to play an important role in removing mis-spliced isoforms and different evolutionary constraints have been observed for NMD-visible (presence of a premature stop codon after retention) and NMD-invisible (absence of a premature stop codon after retention) introns (37), we further divided our 3 positional categories (central, proximal, distal) of introns into NMD-visible or NMD-invisible groups (Fig. 3 A).

First, we observed that the proportion of distal introns is higher in NMD-invisible introns compared to NMD-visible ones, independent of the introduction of a frameshift ( $3 n$ versus non- $3 n$ introns) (Fig. 3 A). We could not observe statistically significant differences between $3 \mathrm{n}$ and non-3n NMD-invisible intron distributions ( $p=0.25, x^{2}$ test), and only a minor significant increase of distal introns at the expense of central introns and proximal introns can be detected between $3 n$ and non-3n NMD-visible introns $\left(p<10^{-}\right.$ ${ }^{6}, X^{2}$ test) (Fig. 3 A). Since no major differences in the intron distribution between $3 \mathrm{n}$ and non-3n introns were observed, we decided to consider only the NMD state for subsequent analysis.

As shown in (37), the intron retention rate is inversely correlated with the gene expression level and is higher for introns that can be detected by the NMD machinery than for those that cannot. In WT cells, both NMD-visible and NMD-invisible introns showed similar retention rates, with higher retention rates for genes with lower expression levels (Fig. 3 B). The retention rate of NMD-visible introns increased 
significantly upon NMD depletion, while it did not in NMD-invisible introns (Fig. 3 B). We extended this analysis to our intron positional categories. As expected, NMD-invisible introns showed similar splicing efficiency for all intron classes in both WT and NMD-depleted cells (Fig. 3 B right panel and Fig. S3 A). Interestingly, we found that the retention rate of NMD-visible introns decreased while the distance of the intron to the closest nucleosome centre increased (Retcentral>RetProximal>RetDistal, Fig. 3 B left and Fig. S3 A), indicating that NMD-visible introns located at the edges of nucleosomes are more efficiently spliced. This can already be observed in a WT background, while in NMD-depleted cells, where nonsense mRNAs are no longer degraded, this difference is much stronger (Fig. 3 B left panel and Fig. $\mathrm{S} 3 \mathrm{~A}$ ). For the lowest-expressed genes, the retention rate of central introns is $57.6 \%$ higher than that of distal introns, and it drops to $38.8 \%$ and $13.9 \%$ for the median and highly-expressed genes, respectively (Fig. $3 \mathrm{~B}$ left panel and Fig. S3 A).

It has been shown that the splicing efficiency of $P$. tetraurelia introns depends on the sequences at the donor and acceptor sites (36). We thus assessed whether this difference in splicing efficiency between our nucleosome-positional classes could be explained by a different distribution of strong donor ( $5^{\prime}$ GTA) and/or strong acceptor (3' TAG) sites (Fig. S3 B) within different intron groups. As expected, NMD-invisible introns were more frequently associated with both strong donors and acceptors whatever the distance of the intron to the closest nucleosome centre (Fig. $3 \mathrm{C}$ ). In contrast, we found a minor increase in the association of distal introns with "weak donor and acceptor" and "strong acceptor only" intron groups compared to central introns, for the NMD-visible introns (Fig. $3 \mathrm{C}$ ). This slight increase was not associated with a higher retention rate for distal introns compared to central introns. Instead, we did observe a reduced retention rate in distal introns (Fig. $3 \mathrm{~B}$ ). We conclude that the reduced retention rate in distal introns is not due to a difference of donor/acceptor signals in this class. 


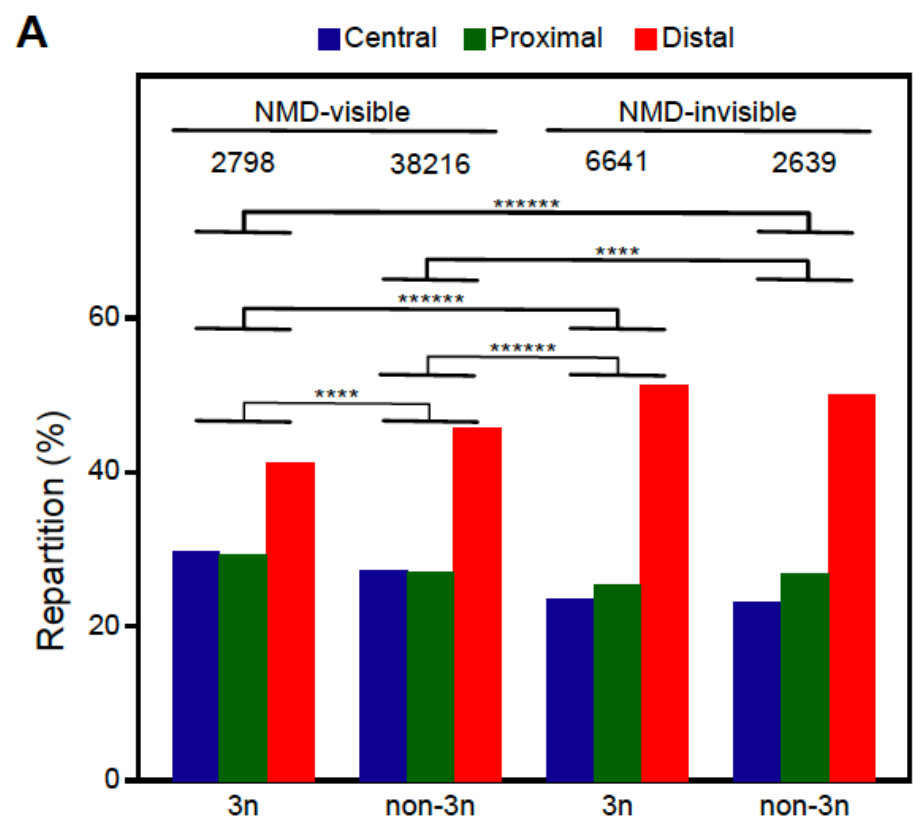

B
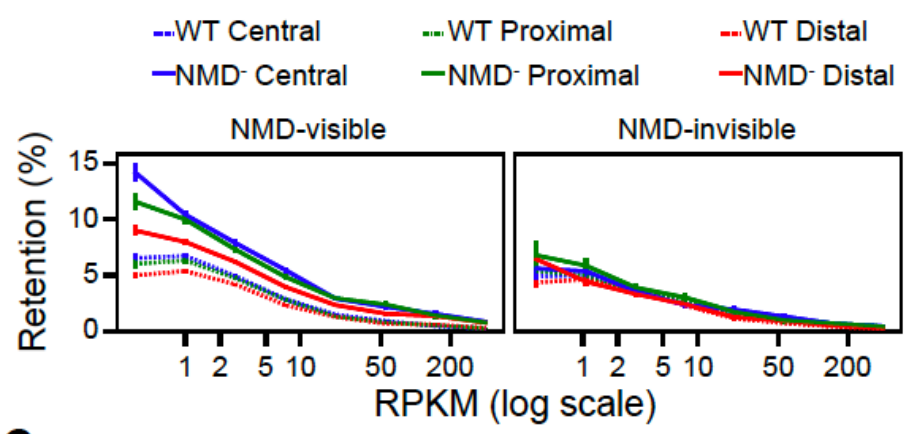

C

Strong donor and acceptor Strong acceptor only

- Strong donor only Weak donor and acceptor

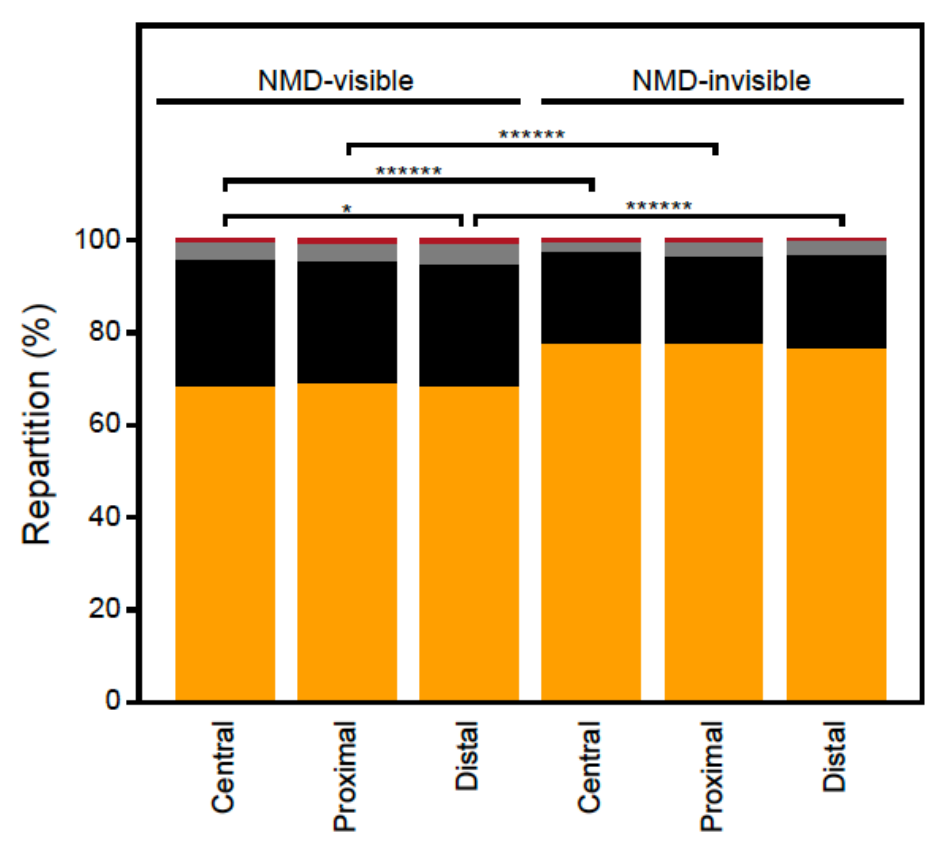


Figure 3. Nucleosome positioning is associated with intron-splicing efficiency. (A) Relative distribution of different classes of introns. Introns are grouped based on their length ( $3 n$ or non-3n) and whether their retention causes a premature stop codon making them visible to the nonsense-mediated decay mechanism (NMD-visible) or not (NMD-invisible). Within each group, introns are classified based on the distance to the closest nucleosome as in Fig. $2 \mathrm{D}$. P-values are calculated using the $\mathrm{X}^{2}$ test and only the significant ones are indicated. (B) The retention rate of introns in WT (dashed lines) and in NMD-depleted (solid lines) cells as a function of gene expression levels. Introns are classified based on their distance to the closest nucleosome as in Fig. $2 \mathrm{D}$ and on whether they are visible to NMD or not. Error bars represent the standard error of the mean. P-values calculated using Mann-Whitley $U$ test, and adjusted with false discovery rate, are displayed in Fig. S3 A. (C) Relative characterization of introns with strong splicing acceptors and/or donors, with the same categories as in Fig. 3 B. P-values are calculated using the $\mathrm{X}^{2}$ test. (P-value $\left.{ }^{*}<0.05,{ }^{* *}<10^{-2},{ }^{* * *}<10^{-3},{ }^{* * * *}<10^{-4},{ }^{* * * * *}<10^{-5},{ }^{* * * * *}<10^{-6}\right)$.

\section{GC content related to nucleosome positioning contributes to intron-splicing efficiency at the edges of nucleosomes}

It is well known that nucleosome positioning is highly associated with GC content: nucleosome centres show higher GC than distal regions (3, 6-9). In $P$. tetraurelia, we observed that NMD-visible introns have a higher GC content than their NMD-invisible counterparts (Fig. 4 A). Moreover, as we would expect, the central introns have the highest GC content followed by proximal and distal introns (Fig. 4 A). We therefore analysed the impact of GC content on intron retention rates. We found a direct correlation between GC percentage and retention rate in NMD-visible introns, yet no statistically significant difference could be observed between different intron groups (Fig. 4 B and Fig. S4 A). This suggests that GC-content anti-correlates with intron splicing efficiency. To further evaluate how different parameters, such as GC content, gene transcription level, and nucleosome positioning (Table S1) affect intron splicing efficiency, we iteratively trained a multivariate regression model as previously described (43), by using different combinations of parameters and retaining only those that were statistically significant (Materials and Methods). The best fitting model $(R=0.6)$, which explains $35 \%$ of variation in intron splicing efficiency was then used to estimate the contribution of each parameter (full list of parameters in Table S1).

The highest contribution came from the level of gene expression that accounts for about $60 \%$ of the model. The GC content of the intron accounted for almost $18 \%$. The length of the intron, its position in the transcript and the total number of introns in a transcript accounted for about $5 \%$ each in the model, followed by the size of the following exons ( $2 \%)$, the GC content of the transcript $(\sim 1 \%)$ and the size of the previous exon $(\sim 1 \%)$. Remarkably, the only parameter relative to the nucleosome positioning retained by the model was the distance to the closest nucleosome centre that accounts for only $0.64 \%$ (Fig. 4 C-D and Table S1). In addition, once we divided the introns into NMD-visible and NMD-invisible, the distance to the closest nucleosome centre was relevant only for the NMD-visible ones where it accounts for $0.67 \%$ of the model (Fig. S4 B-C). We therefore conclude that, the GC content, which is tightly linked to nucleosome positioning, contributes to intron-splicing efficiency. 


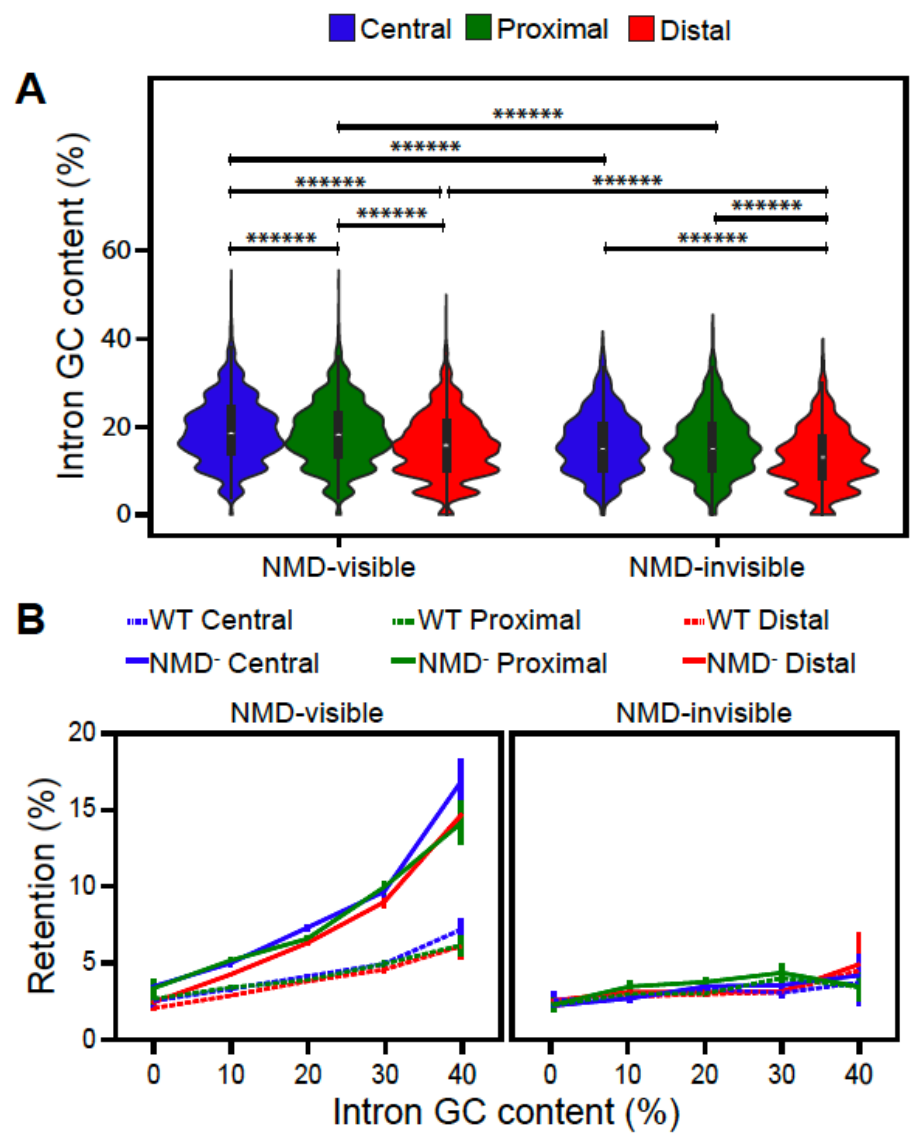

C

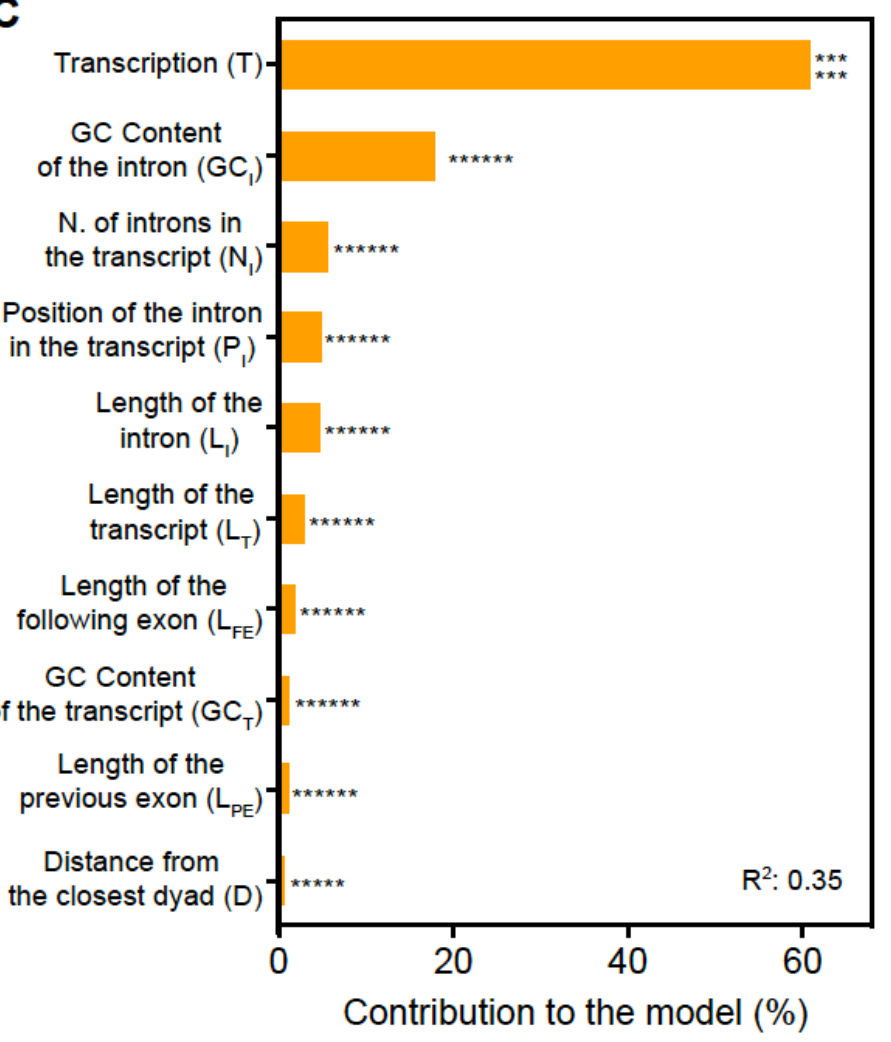

D

$$
\begin{aligned}
\mathrm{e}^{\mathrm{SE}}= & 0.306 \log (\mathrm{T})-0.156 \mathrm{GC}_{\mathrm{I}}+0.062 \mathrm{~N}_{\mathrm{I}}+0.056 \mathrm{P}_{\mathrm{I}} \\
& +0.063 \mathrm{~L}_{\mathrm{I}}+0.082 \mathrm{~L}_{\mathrm{T}}-0.039 \mathrm{~L}_{\mathrm{FE}}-0.022 \mathrm{GC}_{\mathrm{T}} \\
& +0.028 \mathrm{~L}_{\mathrm{PE}}+0.018 \mathrm{D}
\end{aligned}
$$


Figure 4. GC content related to nucleosome positioning contributes to intron-splicing efficiency. (A) GC content (\%) distribution of introns based on the distance to the closest nucleosome centre and NMD visibility. P-values were calculated using the Mann-Whitney $U$ test and adjusted using the false discovery rate. (B) The retention rate of introns in WT and NMD-depleted cells as a function of their GC content (excluded GT and AG dinucleotides at both extremities). Introns are classified based on their distance to the closest nucleosome centre and on whether they are visible or not to NMD. Binning = $10 \%$. Error bars represent the standard error of the mean. P-values calculated using the Mann-Whitney $\mathrm{U}$ test, and adjusted using the false discovery rate, are displayed in Fig. S4 A. (C) Modelling Splicing Efficient (SE) in NMD-depleted cells, showing the parameters used to fit our model, with the contribution of each parameter to the model and their statistical significance (P-Val). (D) The full fitted model in explaining intron splicing efficiency, indicating whether each parameter is positively or negatively correlated with splicing efficiency. The parameters are as indicated in (C). (P-value ${ }^{*}<0.05,{ }^{* *}<10^{-2}$, $\left.{ }^{* * *}<10^{-3},{ }^{* * * *}<10^{-4},{ }^{* \star \star * *}<10^{-5},{ }^{* * * * * *}<10^{-6}\right)$.

\section{DISCUSSION}

We have performed the first nucleosome position profiling in the $P$. tetraurelia MAC genome during vegetative growth. Despite its high AT richness (>75\% AT), the $P$. tetraurelia MAC genome displays a very regular nucleosome positioning pattern as observed in other eukaryotes: NFRs at the TSSs and TTSs, and a regular nucleosome array along genes. Unlike Tetrahymena thermophila, another AT-rich ciliate $(78 \%$ AT) (with an NRL of $199 \mathrm{bp}$ ) (48), the NRL in the $P$. tetraurelia MAC genome presents a

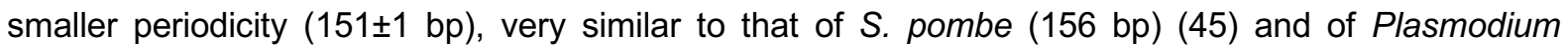
falciparum (155 bp) (>80\%AT) $(49,50)$. This short NRL means that the naked "linker" DNA between nucleosomes in Paramecium is extremely small (only a few bp) compared to that of most other eukaryotic genomes, which is at least tens of bp or even larger (51). A negative correlation between the H1/core-histone ratio and NRL has been previously reported (52-54). Importantly, for the three eukaryotes with the smallest NRL, P. tetraurelia, P. falciparum and S. pombe, no orthologue of histone $\mathrm{H} 1$ has been identified so far. This strongly suggests that the absence of $\mathrm{H} 1$ might contribute to the extremely short NRL observed in Paramecium chromatin organization in the somatic MAC genome.

In yeast and human, actively transcribed genes tend to have shorter NRL than transcriptionally inactive genes, partially due to the binding of $\mathrm{H} 1$ generating inaccessible chromatin at inactive genes $(47,55$, 56). With the separation of the germline MIC and the somatic MAC genomes in two distinct nuclei, the Paramecium MAC genome is very compact. Indeed, $>80 \%$ of the MAC is covered by annotated genes and $65 \%$ of the coding genes are expressed (covered by at least 1 RPKM) during vegetative growth $(34,42)$, which might explain the extremely short length and narrow distribution of NRL. A significant difference in the nucleosome organization between MAC and MIC genomes has been reported for $T$. thermophila (57). How nucleosomes are organized in the Paramecium MIC genome is unknown. At each sexual cycle of Paramecium, the parental MAC is destroyed and the new MIC and MAC are generated from the parental germline MIC (35). During new MAC development, an estimated $\sim 25 \%$ of the germline DNA is eliminated during massive genome rearrangements (58). A large amount of 
extremely short (26 to $\sim 1000 \mathrm{bp}$ ) non-coding germline sequences, called IESs (Internal Eliminated Sequences), need to be precisely excised to correctly assembly functional genes in the new MAC genome of Paramecium species (59). How nucleosome positioning is organized in the germline MIC genome relative to IESs and whether nucleosome positioning and/or GC content might play a role in IES excision are open questions $(60,61)$.

In multicellular eukaryotes, long introns are recognized through exon definition and nucleosomes positioned along exons might contribute to the exon-intron architecture, possibly pointing to a function in exon definition (20-25). By contrast, short introns are recognized through intron definition. With an average length of $25 \mathrm{nt}$, introns of $P$. tetraurelia are among the shortest reported in eukaryotes (36). The large number of introns $(>90,000)$ are associated with weak splicing signals. In the current study, we examined the role of nucleosome positioning in intron splicing. We found a regular nucleosome array associated with intron positions within genes, with exons wrapped around nucleosomes and introns frequently located at the edge of nucleosomes. By using the accurate splicing efficiency data obtained from NMD-depleted cells (37), we performed a thorough investigation on the effect of nucleosome positioning on splicing efficiency. We showed that the NMD-visible introns located at the edge of nucleosomes display higher splicing efficiency than those at the nucleosome centres. However, we found that this higher splicing efficiency is due to the fact that the introns located at the edges of nucleosomes display lower GC content. Our multiple regression analysis indicated that the nucleosome positioning did not show any contribution to the intron splicing efficiency (Fig. $4 \mathrm{C}$ ). Our results strongly support that the GC content, rather than the nucleosome positioning, directly influences intron splicing efficiency in Paramecium, which may pave the way to future mechanistic studies to decipher how GC content may impinge on intron splicing efficiency. Whether the effect of GC content and nucleosome positioning on intron splicing efficiency observed in Paramecium can be extended to other eukaryotes remains an open question.

Interestingly, we also observed that during evolution, nucleosome positioning has been displaced relative to introns, so that the AT-rich intron sequences are frequently located at the edge of nucleosomes (Fig. 4 A). Although both NMD-visible and NMD-invisible introns present a higher proportion of distal positions, NMD-invisible introns show a significantly higher proportion ( $51 \%$ for $3 n$ and $49 \%$ for non- $3 n$ introns) than NMD-visible introns ( $41 \%$ for $3 n$ and $46 \%$ for non- $3 n$ introns) (Fig. 3 A). This strongly suggests that the NMD-invisible introns not located at the AT-rich nucleosome edges, whose retention in transcripts cannot be cleaned up by the NMD pathway, are counter-selected during evolution. Whether introns in Paramecium might play a functional role is still unclear. These introns do not seem to contribute to alternative splicing to generate protein diversity or to encode ncRNAs as in other eukaryotic genomes (62-64). Due to their extremely small sizes, these introns are unlikely to play a role in regulating the transcription rate as suggested in recent publications (65-67). How such a large number of tiny introns in Paramecium is maintained during evolution and how these introns can be efficiently spliced need to be further investigated. 


\section{DATA AVAILABILITY}

The gene annotations and transcription data are available at ParameciumDB (https://paramecium.i2bc.paris-saclay.fr/). The customized script and Jupiter notebooks used for this study are available on the GitHub page (https://github.com/CL-CHEN-Lab/Nucleosome).

\section{ACCESSION NUMBERS}

MNase-seq datasets are deposited in the ENA under the Project Accession PRJEB39679 (68).

\section{FUNDING}

This work was supported by Centre National de la Recherche Scientifique, the Agence Nationale pour la Recherche (ANR) [project "GENOMAC" ANR-10-BLAN-1603 to M.B., C.T., C.L.C, S.D.]; [project "LaMarque" ANR-18-CE12-0005; to S.D., M.B.] and [project "POLYCHROME" ANR-19-CE12-0015 to S.D. and O.A.]; the LABEX Who Am I? to S.D. (ANR-11-LABX-0071; ANR-11-IDEX-0005-02). The salary of S.G. was provided by the ATIP-Avenir program from CNRS and Plan Cancer (C.L.C.). Funding for open access charge: "LaMarque" ANR-18-CE12-0005.

\section{AUTHOR CONTRIBUTIONS}

LS, MB, CT, CLC and SD conceived and planned the study. MM, FG and SD conducted the experiments. SG, MW, OA and CLC performed the bioinformatics analyses. CT and CC supervised the bioinformatics analyses. SG, CLC and SD wrote the manuscript, and all the authors reviewed it.

\section{CONFLICT OF INTEREST}

The authors declare no competing interest.

\section{ACKNOWLEDGEMENTS}

The authors would like to thank Laurent Duret for useful suggestions and discussion, Laurent Duret and Eric Meyer for sharing with us the NMD data, and to acknowledge the high-throughput sequencing facility of $\mathrm{I} 2 \mathrm{BC}$ for its sequencing and bioinformatics expertise.

\section{REFERENCES}

1. Luger, K., Mä Der, A.W., Richmond, R.K., Sargent, D.F. and Richmond, T.J. (1997) Crystal structure of the nucleosome core particle at $2.8 \AA$ resolution. Nature, 389, 251-260.

2. Parmar, J.J. and Padinhateeri, R. (2020) Nucleosome positioning and chromatin organization. Curr. Opin. Struct. Biol., 64, 111-118.

3. Tillo, D. and Hughes, T.R. (2009) G+C content dominates intrinsic nucleosome occupancy. BMC Bioinformatics, 10.

4. Tillo, D., Kaplan, N., Moore, I.K., Fondufe-Mittendorf, Y. and Gossett, A.J. (2010) High Nucleosome Occupancy Is Encoded at Human Regulatory Sequences. PLoS One, 5, 9129.

5. Segal, E., Fondufe-Mittendorf, Y., Chen, L., Thåström, A., Field, Y., Moore, I.K., Wang, J.P.Z. and Widom, J. (2006) A genomic code for nucleosome positioning. Nature, 442, 772-778. 
6. Lorch, Y., Maier-Davis, B. and Kornberg, R.D. (2014) Role of DNA sequence in chromatin remodeling and the formation of nucleosome-free regions. Genes Dev., 28, 2492-2497.

7. Lyer, V. and Struhl, K. (1995) Poly(dA:dT), a ubiquitous promoter element that stimulates transcription via its intrinsic DNA structure. EMBO J., 14, 2570-2579.

8. Peckham, H.E., Thurman, R.E., Fu, Y., Stamatoyannopoulos, J.A., Noble, W.S., Struhl, K. and Weng, Z. (2007) Nucleosome positioning signals in genomic DNA. Genome Res., 17, 1170-1177.

9. Vaillant, C., Palmeira, L., Chevereau, G., Audit, B., d'Aubenton-Carafa, Y., Thermes, C. and Arneodo, A. (2010) A novel strategy of transcription regulation by intragenic nucleosome ordering. Genome Res., 20, 59-67.

10. Bartholomew, B. (2014) Regulating the chromatin landscape: Structural and mechanistic perspectives. Annu. Rev. Biochem., 83, 671-696.

11. Prendergast, J.G.D. and Semple, C.A.M. (2011) Widespread signatures of recent selection linked to nucleosome positioning in the human lineage. Genome Res., 21, 1777-1787.

12. Kornberg, R.D. and Lorch, Y. (2020) Primary Role of the Nucleosome. Mol. Cell, 79, 371-375.

13. Jiang, C. and Pugh, B.F. (2009) Nucleosome positioning and gene regulation: Advances through genomics. Nat. Rev. Genet., 10, 161-172.

14. Bernstein, B.E., Liu, C.L., Humphrey, E.L., Perlstein, E.O. and Schreiber, S.L. (2004) Global nucleosome occupancy in yeast. Genome Biol., 5.

15. Yuan, G.C., Liu, Y.J., Dion, M.F., Slack, M.D., Wu, L.F., Altschuler, S.J. and Rando, O.J. (2005) Genome-scale identification of nucleosome positions in S. cerevisiae. Science (80-. )., 309, 626630.

16. Fan, X., Moqtaderi, Z., Jin, Y., Zhang, Y., Liu, X.S. and Struhl, K. (2010) Nucleosome depletion at yeast terminators is not intrinsic and can occur by a transcriptional mechanism linked to 3 '-end formation. Proc. Natl. Acad. Sci. U. S. A., 107, 17945-17950.

17. Chereji, Ř. V., Kan, T.W., Grudniewska, M.K., Romashchenko, A. V., Berezikov, E., Zhimulev, I.F., Guryev, V., Morozov, A. V. and Moshkin, Y.M. (2016) Genome-wide profiling of nucleosome sensitivity and chromatin accessibility in Drosophila melanogaster. Nucleic Acids Res., 44, 10361051.

18. Beshnova, D.A., Cherstvy, A.G., Vainshtein, Y. and Teif, V.B. (2014) Regulation of the Nucleosome Repeat Length In Vivo by the DNA Sequence, Protein Concentrations and Long-Range Interactions. PLoS Comput. Biol., 10, e1003698.

19. Allan, J., Fraser, R.M., Owen-Hughes, T., Docherty, K. and Singh, V. (2013) A comparison of in vitro nucleosome positioning mapped with chicken, frog and a variety of yeast core histones. $J$. Mol. Biol., 425, 4206-4222.

20. Andersson, R., Enroth, S., Rada-Iglesias, A., Wadelius, C. and Komorowski, J. (2009) Nucleosomes are well positioned in exons and carry characteristic histone modifications. Genome Res., 19, 1732-41.

21. Schwartz, S., Meshorer, E. and Ast, G. (2009) Chromatin organization marks exon-intron structure. Nat. Struct. Mol. Biol., 16, 990-5.

22. Tilgner, H., Nikolaou, C., Althammer, S., Sammeth, M., Beato, M., Valcárcel, J. and Guigó, R. (2009) 
Nucleosome positioning as a determinant of exon recognition. Nat. Struct. Mol. Biol., 16, 9961001.

23. Nahkuri, S., Taft, R.J. and Mattick, J.S. (2009) Nucleosomes are preferentially positioned at exons in somatic and sperm cells. Cell Cycle, 8, 3420-3424.

24. Spies, N., Nielsen, C.B., Padgett, R.A. and Burge, C.B. (2009) Biased Chromatin Signatures around Polyadenylation Sites and Exons. Mol. Cell, 36, 245-254.

25. Iannone, C., Pohl, A., Papasaikas, P., Soronellas, D., Vicent, G.P., Beato, M. and ValcáRcel, J. (2015) Relationship between nucleosome positioning and progesterone-induced alternative splicing in breast cancer cells. RNA, 21, 360-74.

26. Jonkers, I., Kwak, H. and Lis, J.T. (2014) Genome-wide dynamics of Pol II elongation and its interplay with promoter proximal pausing, chromatin, and exons. Elife, 2014, e02407.

27. Wilhelm, B.T., Marguerat, S., Aligianni, S., Codlin, S., Watt, S. and Bähler, J. (2011) Differential patterns of intronic and exonic DNA regions with respect to RNA polymerase II occupancy, nucleosome density and H3K36me3 marking in fission yeast. Genome Biol., 12, R82.

28. Brody, Y., Neufeld, N., Bieberstein, N., Causse, S.Z., Böhnlein, E.M., Neugebauer, K.M., Darzacq, X. and Shav-Tal, Y. (2011) The in vivo kinetics of RNA polymerase II elongation during cotranscriptional splicing. PLoS Biol., 9.

29. Herzel, L., Ottoz, D.S.M., Alpert, T. and Neugebauer, K.M. (2017) Splicing and transcription touch base: Co-transcriptional spliceosome assembly and function. Nat. Rev. Mol. Cell Biol., 18, 637650.

30. Amit, M., Donyo, M., Hollander, D., Goren, A., Kim, E., Gelfman, S., Lev-Maor, G., Burstein, D., Schwartz, S., Postolsky, B., et al. (2012) Differential GC content between exons and introns establishes distinct strategies of splice-site recognition. Cell Rep., 1, 543-56.

31. Gelfman, S., Cohen, N., Yearim, A. and Ast, G. (2013) DNA-methylation effect on cotranscriptional splicing is dependent on GC architecture of the exon-intron structure. Genome Res., 23, 789-99.

32. Lykke-Andersen, S. and Jensen, T.H. (2015) Nonsense-mediated mRNA decay: An intricate machinery that shapes transcriptomes. Nat. Rev. Mol. Cell Biol., 16, 665-677.

33. Kurosaki, T., Popp, M.W. and Maquat, L.E. (2019) Quality and quantity control of gene expression by nonsense-mediated mRNA decay. Nat. Rev. Mol. Cell Biol., 20, 406-420.

34. Aury, J.-M., Jaillon, O., Duret, L., Noel, B., Jubin, C., Porcel, B.M., Ségurens, B., Daubin, V., Anthouard, V., Aiach, N., et al. (2006) Global trends of whole-genome duplications revealed by the ciliate Paramecium tetraurelia. Nature, 444, 171-178.

35. Betermier, M. and Duharcourt, S. (2014) Programmed Rearrangement in Ciliates: Paramecium. Microbiol. Spectr., 2.

36. Jaillon, O., Bouhouche, K., Gout, J.-F., Aury, J.-M., Noel, B., Saudemont, B., Nowacki, M., Serrano, V., Porcel, B.M., Ségurens, B., et al. (2008) Translational control of intron splicing in eukaryotes. Nature, 451, 359-62.

37. Saudemont, B., Popa, A., Parmley, J.L., Rocher, V., Blugeon, C., Necsulea, A., Meyer, E. and Duret, L. (2017) The fitness cost of mis-splicing is the main determinant of alternative splicing patterns. Genome Biol., 18, 208. 
38. Beisson, J., Bétermier, M., Bré, M.H., Cohen, J., Duharcourt, S., Duret, L., Kung, C., Malinsky, S., Meyer, E., Preer, J.R., et al. (2010) Maintaining clonal paramecium tetraurelia cell lines of controlled age through daily reisolation. Cold Spring Harb. Protoc., 5, pdb.prot5361.

39. Beisson, J., Bétermier, M., Bré, M.H., Cohen, J., Duharcourt, S., Duret, L., Kung, C., Malinsky, S., Meyer, E., Preer, J.R., et al. (2010) Mass culture of paramecium tetraurelia. Cold Spring Harb. Protoc., 5, pdb.prot5362.

40. Arnaiz, O., Mathy, N., Baudry, C., Malinsky, S., Aury, J.-M., Denby Wilkes, C., Garnier, O., Labadie, K., Lauderdale, B.E., Le Mouël, A., et al. (2012) The Paramecium germline genome provides a niche for intragenic parasitic DNA: evolutionary dynamics of internal eliminated sequences. PLoS Genet., 8, e1002984.

41. Arnaiz, O., Meyer, E. and Sperling, L. (2019) ParameciumDB 2019: integrating genomic data across the genus for functional and evolutionary biology. Nucleic Acids Res., 10.1093/nar/gkz948.

42. Arnaiz, O., Van Dijk, E., Bétermier, M., Lhuillier-Akakpo, M., de Vanssay, A., Duharcourt, S., Sallet, E., Gouzy, J. and Sperling, L. (2017) Improved methods and resources for paramecium genomics: transcription units, gene annotation and gene expression. BMC Genomics, 18, 483.

43. Chen, C.-L., Rappailles, A., Duquenne, L., Huvet, M., Guilbaud, G., Farinelli, L., Audit, B., D'Aubenton-Carafa, Y., Arneodo, A., Hyrien, O., et al. (2010) Impact of replication timing on nonCpG and CpG substitution rates in mammalian genomes. Genome Res., 20, 447-57.

44. Chereji, R. V., Ocampo, J. and Clark, D.J. (2017) MNase-Sensitive Complexes in Yeast: Nucleosomes and Non-histone Barriers. Mol. Cell, 65, 565-577.e3.

45. Godde, J.S. and Widom, J. (1992) Chromatin structure of Schizosaccharomyces pombe. A nucleosome repeat length that is shorter than the chromatosomal DNA length. J. Mol. Biol., 226, 1009-1025.

46. van Holde, K.E. (1989) Chromatin Springer New York, New York, NY, NY.

47. Valouev, A., Johnson, S.M., Boyd, S.D., Smith, C.L., Fire, A.Z. and Sidow, A. (2011) Determinants of nucleosome organization in primary human cells. Nature, 474, 516-20.

48. Beh, L.Y., Müller, M.M., Muir, T.W., Kaplan, N. and Landweber, L.F. (2015) DNA-guided establishment of nucleosome patterns within coding regions of a eukaryotic genome. Genome Res., 25, 1727-38.

49. Kensche, P.R., Hoeijmakers, W.A.M., Toenhake, C.G., Bras, M., Chappell, L., Berriman, M. and Bártfai, R. (2015) The nucleosome landscape of Plasmodium falciparum reveals chromatin architecture and dynamics of regulatory sequences. Nucleic Acids Res., 44, 2110-24.

50. Silberhorn, E., Schwartz, U., Löffler, P., Schmitz, S., Symelka, A., de Koning-Ward, T., Merkl, R. and Längst, G. (2016) Plasmodium falciparum Nucleosomes Exhibit Reduced Stability and Lost Sequence Dependent Nucleosome Positioning. PLOS Pathog., 12, e1006080.

51. Arceci, R.J. and Gross, P.R. (1980) Sea urchin sperm chromatin structure as probed by pancreatic DNase I: Evidence for a novel cutting periodicity. Dev. Biol., 80, 210-224.

52. Fan, Y., Nikitina, T., Morin-Kensicki, E.M., Zhao, J., Magnuson, T.R., Woodcock, C.L. and Skoultchi, A.I. (2003) H1 Linker Histones Are Essential for Mouse Development and Affect Nucleosome Spacing In Vivo. Mol. Cell. Biol., 23, 4559-4572. 
53. Fan, Y., Nikitina, T., Zhao, J., Fleury, T.J., Bhattacharyya, R., Bouhassira, E.E., Stein, A., Woodcock, C.L. and Skoultchi, A.I. (2005) Histone H1 depletion in mammals alters global chromatin structure but causes specific changes in gene regulation. Cell, 123, 1199-1212.

54. Woodcock, C.L., Skoultchi, A.I. and Fan, Y. (2006) Role of linker histone in chromatin structure and function: H1 stoichiometry and nucleosome repeat length. Chromosom. Res., 14, 17-25.

55. Correll, S.J., Schubert, M.H. and Grigoryev, S.A. (2012) Short nucleosome repeats impose rotational modulations on chromatin fibre folding. EMBO J., 31, 2416-2426.

56. Barbier, J., Vaillant, C., Volff, J.-N., Brunet, F. and Audit, B. (2021) Coupling Between SequenceMediated Nucleosome Organization and Genome Evolution. 10.20944/PREPRINTS202105.0166.V1.

57. Xiong, J., Gao, S., Dui, W., Yang, W., Chen, X., Taverna, S.D., Pearlman, R.E., Ashlock, W., Miao, W. and Liu, Y. (2016) Dissecting relative contributions of cis-and trans-determinants to nucleosome distribution by comparing Tetrahymena macronuclear and micronuclear chromatin. Nucleic Acids Res., 44, 10091-10105.

58. Guérin, F., Arnaiz, O., Boggetto, N., Denby Wilkes, C., Meyer, E., Sperling, L. and Duharcourt, S. (2017) Flow cytometry sorting of nuclei enables the first global characterization of Paramecium germline DNA and transposable elements. BMC Genomics, 18, 327.

59. Sellis, D., Guérin, F., Arnaiz, O., Pett, W., Lerat, E., Boggetto, N., Krenek, S., Berendonk, T., Couloux, A., Aury, J.-M., et al. (2021) Massive colonization of protein-coding exons by selfish genetic elements in Paramecium germline genomes. PLOS Biol., 19, e3001309.

60. Coyne, R.S., Lhuillier-Akakpo, M. and Duharcourt, S. (2012) RNA-guided DNA rearrangements in ciliates: is the best genome defence a good offence? Biol. cell, 104, 309-25.

61. Lhuillier-Akakpo, M., Frapporti, A., Denby Wilkes, C., Matelot, M., Vervoort, M., Sperling, L. and Duharcourt, S. (2014) Local effect of enhancer of zeste-like reveals cooperation of epigenetic and cis-acting determinants for zygotic genome rearrangements. PLoS Genet., 10, e1004665.

62. Lee, Y. and Rio, D.C. (2015) Mechanisms and regulation of alternative Pre-mRNA splicing. Annu. Rev. Biochem., 84, 291-323.

63. Chen, C.L., Liang, D., Zhou, H., Zhuo, M., Chen, Y.Q. and Qu, L.H. (2003) The high diversity of snoRNAs in plants: Identification and comparative study of 120 snoRNA genes from Oryza sativa. Nucleic Acids Res., 31, 2601-2613.

64. Ruby, J.G., Jan, C.H. and Bartel, D.P. (2007) Intronic microRNA precursors that bypass Drosha processing. Nature, 448, 83-86.

65. Fong, N., Kim, H., Zhou, Y., Ji, X., Qiu, J., Saldi, T., Diener, K., Jones, K., Fu, X.D. and Bentley, D.L. (2014) Pre-mRNA splicing is facilitated by an optimal RNA polymerase II elongation rate. Genes Dev., 28, 2663-2676.

66. Aslanzadeh, V., Huang, Y., Sanguinetti, G. and Beggs, J.D. (2018) Transcription rate strongly affects splicing fidelity and cotranscriptionality in budding yeast. Genome Res., 28, 203-213.

67. Alexander, R.D., Innocente, S.A., Barrass, J.D. and Beggs, J.D. (2010) Splicing-Dependent RNA polymerase pausing in yeast. Mol. Cell, 40, 582-593.

68. Hardy, A., Matelot, M., Touzeau, A., Klopp, C., Lopez-Roques, C., Duharcourt, S. and Defrance, M. 
bioRxiv preprint doi: https://doi.org/10.1101/2021.08.05.455221: this version posted Auqust 6.2021. The copyriaht holder for this preprint (which was not certified by peer review) is the author/funder, who has granted bioRxiv a license to display the preprint in perpetuity. It is made available under aCC-BY-NC-ND 4.0 International license.

(2021) DNAModAnnot: a R toolbox for DNA modification filtering and annotation. Bioinformatics, 10.1093/bioinformatics/btab032. 